BESANCON

SUR LES DENOMINATEURS DES FONCTIONS ZÊETA PARTIELLES 


\title{
Sur les dénominateurs des fonctions zêta partielles
}

\author{
Georges GRAS
}

Résumé : Soit $k$ un corps de nombres totalement réel. Soient $S_{0}$ un ensemble fini de nombres premiers, $S$ l'ensemble des places de $k$ au-dessus de $S_{0} \cup\{\infty\}$, et $F / k$ une extension abélienne finie non ramifiée en dehors de $S$. Alors pour tout $p \in S_{0}$, les valuations $p$-adiques des dénominateurs des fonctions zêta partielles $\zeta_{S, F}\left(\sigma_{F}, 1-n\right), \sigma_{F} \in \mathrm{Gal}(F / k), n \geq 1$, sont données explicitement. Il en résulte, en tout nombre premier, des critères analytiques pour la conjecture de Leopoldt dans $k$ qui précisent ceux donnés par J.-P. Serre.

\section{0) Introduction et résultat principal :}

Soit $k$ un corps de nombres totalement réel. Soit $S_{0}$ un ensemble fini de nombres premiers ; l'ensemble $S$ des places de $k$ au-dessus de $S_{0} \cup\{\infty\}$ contient donc les $[k: \mathbb{Q}]$ places à l'infini de $k$; soient alors $k_{S}$ l'extension abélienne $S$-ramifiée maximale de $k$ et $G_{S}=\mathrm{Gal}\left(k_{S} / k\right)$. Soit $F$ une extension finie de $k$ dans $k_{S}$ et soit $G_{F}=\mathrm{Gal}(F / k)$. La fonction zêta partielle $\zeta_{S, F}$ est définie, pour chaque $\sigma_{F} \in G_{F}$, par prolongement analytique de

$$
\zeta_{S, F}\left(\sigma_{F}, s\right)=\sum_{\mathfrak{A}} N \mathfrak{A}^{-s}, s \in \mathbb{C}, \operatorname{Ré}(s)>1,
$$

où la somme est prise sur les idéaux entiers $\mathfrak{A}$ de $k$ étrangers à $S$ et tels que le symbole d'Artin $\left(\frac{F / k}{\mathfrak{A}}\right)$ soit égal à $\sigma_{F}$. D'après les résultats de H. Klingen et C.L. Siegel (cf. [Si]), on a $\zeta_{S, F}\left(\sigma_{F}, 1-n\right) \in \mathbb{Q}$ pour tout $n \geq 1$. Le résultat suivant sur les dénominateurs des fonctions zêta partielles, après avoir été conjecturé par J.-P. Serre, a été démontré par P. Deligne et K. Ribet [D-R], via les formes modulaires, ainsi que par D. Barsky [B] et P. Cassou-Noguès [CN], via la théorie de Shintani :

(0.0) Théorème : Soit $w_{n}(F), n \geq 1$, le plus grand entier $m$ tel que $\left(\operatorname{Gal}\left(F\left(\mu_{m}\right) / F\right)\right)^{n}=0$, où $\mu_{m}$ désigne le groupe des racines $m$-ièmes de l'unité. Alors $w_{n}(F) \zeta_{S, F}\left(\sigma_{F}, 1-n\right) \in \mathbb{Z}$ pour tout $n \geq 1$.

Ce résultat donne la meilleure divisibilité générale pour les dénominateurs des fonctions zêta partielles, mais pour $p \in S_{0}$, la théorie des fonctions $L$-adiques permet d'interpréter les éléments de Stickelberger d'ordre $n \geq 1$,

$$
S t_{S, F}^{(n)}=\sum_{\sigma_{F} \in G_{F}} \zeta_{S, F}\left(\sigma_{F}, 1-n\right) \sigma_{F}^{-1}
$$

comme les restrictions à $F$ de $\mathbb{Z}_{p}$-distributions sur $G_{S}$ issues de la pseudo-mesure de Deligne-Ribet $D R_{S}$ associée à $S$ (cf. (1.3.1)), et pour laquelle on connait le terme polaire, grâce aux résultats de J.-P. Serre [S] et P. Colmez [C]. Il est alors possible de calculer 
exactement les $p$-parties des dénominateurs en fonction des invariants standard de $k$ que nous précisons par les quelques notations suivantes :

\section{(0.2) Notations :}

(i) Pour tout nombre premier $p$, on désigne par $S_{p}$ l'ensemble des idéaux premiers de $k$ au-dessus de $p$; de même on désigne par $S_{\infty}$ l'ensemble des $[k: Q]$ places à l'infini de $k$. Pour $p$ premier on désigne par $\mathbb{Q}_{\infty}$ la $\mathbf{Z}_{\boldsymbol{p}}$-extension cyclotomique de $k$ et on pose $\left[k_{\cap} \mathbb{Q}_{\infty}: \mathbb{Q}\right]=p^{n_{p}(k)}, n_{p}(k) \geq 0$. On pose enfin $q=p($ resp. 4) si $p \neq 2$ (resp. $p=2$ ).

(ii) Lorsque $\mathfrak{L}$ est un idéal premier de $k, N \mathfrak{L}$ désigne sa norme absolue, et lorsque $\mathfrak{L}$ est une place à l'infini (réelle) de $k$, on pose $N \mathfrak{L}=-1$.

(iii) On désigne par $D(k)$ et $h(k)$ le discriminant et le nombre de classes de $k$, et pour tout nombre premier $p$, on désigne par $R_{p}(k)$ le régulateur $p$-adique de $k$. On pose

$$
\begin{aligned}
\rho_{S, p}(k) & =\frac{h(k) R_{p}(k)}{2 \sqrt{D(k)}} \prod_{\mathfrak{L} \in S}\left(1-\frac{1}{N \mathfrak{L}}\right) \\
& \sim 2^{[k: \mathbb{Q}]} \frac{h(k) R_{p}(k)}{2 \sqrt{D(k)}} \prod_{\mathfrak{p} \in S_{\mathfrak{p}}} N \mathfrak{p}^{-1} \prod_{\mathfrak{L} \in S-S_{p} \cup S_{\infty}}(N \mathfrak{L}-1),
\end{aligned}
$$

où $\sim$ désigne la relation d'équivalence modulo $\mathbf{Z}_{p}^{*}$ dans $\mathbb{Q}_{p}^{*}$.

D'après P. Colmez $[\mathrm{C}]$, on sait que $\rho_{S, p}(k)$ est le résidu en $s=1$ de la fonction zêta $p$-adique $\zeta_{S, p}(k, s)=\zeta_{p}(k, s) \prod_{\mathfrak{L} \in S-S_{p \cup} S_{\infty}}\left(1-N \mathfrak{L}^{-s}\right)$, où $\zeta_{p}(k, s)$ est la fonction zêta $p$-adique de $k$, et que la $p$-partie de

$$
a_{S, p}(k)=q p^{n_{p}(k)} \rho_{S, p}(k)
$$

est, lorsque $R_{p}(k) \neq 0$, le nombre d'éléments du sous-groupe de torsion du $p$-sousgroupe de Sylow de $G_{S}$ (cf. [S, (2.8), (2.9)]).

(iv) On désigne enfin par $\omega$ le caractère de Teichmuller pour $p$ sur $G_{S}$.

Le résultat principal est alors le suivant :

(0.3) Théorème : Soit $F$ une extension abélienne finie $S$-ramifiée de $k$, et soit $n$ un entier $\geq 1$. Alors pour tout $p \in S_{0}$, les valuations $p$-adiques $V_{p}(S, F, 1-n)$ des dénominateurs des rationnels $\zeta_{S, F}\left(\sigma_{F}, 1-n\right), \sigma_{F} \in \operatorname{Gal}(F / k)$, sont indépendantes de $\sigma_{F}$ et sont les suivantes :

(i) $\omega^{n}$ est caractère de $F$ : on a

$$
V_{p}(S, F, 1-n)=\operatorname{Max}\left(0, v_{p}\left(\frac{[F: k] n}{\rho_{S, p}(k)}\right)\right)
$$

(ii) $\omega^{n}$ n'est pas caractère de $F$ : on a $V_{p}(S, F, 1-n)=0$ sauf dans le cas particulier où $p=2, n \equiv 1 \bmod 2$ et $F_{n} k\left(\mu_{2 \infty}\right)$ est imaginaire, cas pour lequel on a :

$$
V_{2}(S, F, 1-n)=\operatorname{Max}\left(0, v_{2}\left(\frac{\left[F: F_{n} k\left(\mu_{2}\right)\right]}{2^{n_{2}(k)} \rho_{S, 2}(k)}\right)\right) \text {. }
$$




\section{(0.4) Remarques :}

(i) Nous avons, dans l'énoncé du théorème, supposé implicitement que la conjecture de Leopoldt est vérifiée pour $p \in S_{0}$ dans $k$ (i.e. $\rho_{S, p}(k) \neq 0$ ), car on sait d'après $[S]$ que, dans le cas contraire, les $\zeta_{S, F}\left(\sigma_{F}, 1-n\right)$ sont toutes $p$-entières. Se reporter aussi aux énoncés (2.12.1') et (2.12.2') qui précisent le résultat précédent.

(ii) Si l'on se donne un nombre premier $p$ arbitraire, on peut, en considérant $S_{0}=$ $\{p\}$ et en choisissant $F \subset k_{S}$ convenable, obtenir des critères d'exactitude de la conjecture de Leopoldt pour $p$ dans $k$, en termes de fonctions zêta partielles en $s \in-\mathbf{N}$ (cf. théorèmes (3.1), (3.2)).

(iii) Ces résultats redonnent et précisent certains de ceux obtenus indépendamment par M. Kolster $[\mathrm{K}]$ au moyen de l'étude de la $K$-théorie étale ; voir également les résultats de $\mathrm{T}$. Nguyen Quang Do $[\mathrm{N}]$ sur les noyaux sauvages étales d'ordres supérieurs.

\section{1) $\boldsymbol{Z}_{p}$-distributions de Stickelberger :}

Soit donc $p \in S_{0}$; rappelons l'essentiel des définitions liées aux questions de mesures $p$ adiques sur $G_{S}=\mathrm{Gal}\left(k_{S} / k\right)$, et qui font intervenir, de manière essentielle, le fait que $S$ contient $S_{p}$ :

\section{(1.1) Définitions :}

(i) Soit $\wedge_{S}$ (resp. $\Delta_{S}, \Delta_{S}^{\prime}$ ) la $\mathbb{Z}_{p}$-algèbre topologique des $\mathbb{Z}_{p}$-mesures sur $G_{S}$, égale par définition $\underset{F}{\lim } \mathbb{Z}_{p}\left[G_{F}\right], k \subseteq F \subset k_{S},[F: k]<\infty$, où $G_{F}=\mathrm{Gal}(F / k)$ (resp. son anneau total des fractions, puis la sous-algèbre des éléments $\frac{\nu}{\delta} \in \Delta_{S}, \nu, \delta \in \wedge_{S}, \delta$ non nul et non diviseur de 0 , pour lesquels les composantes $\delta_{F}$ sont aussi non nulles et non diviseurs de 0 dans $\mathbf{Z}_{p}\left[G_{F}\right]$, pour tout $F$ ). Les éléments de $\Delta_{S}$ sont appelés les $\mathbf{Z}_{\boldsymbol{p}}$-distributions sur $G_{S}$; parmi elles on distingue les $\mathbf{Z}_{\boldsymbol{p}}$-pseudo-mesures $\lambda$ qui sont telles que $(1-\sigma) \lambda \in \wedge_{S}$ pour tout $\sigma \in G_{S}$ (cf. [S]).

(ii) Pour toute $\mathbf{Z}_{p}$-mesure $\nu \in \wedge_{S}$, on désigne donc par $\nu_{F}$ sa composante sur $\mathbf{Z}_{p}\left[G_{F}\right]$; l'application $\wedge_{S} \rightarrow \mathbb{Z}_{p}\left[G_{F}\right]$, qui à $\nu$ associe $\nu_{F}$, se prolonge en une application de $\Delta_{S}^{\prime}$ dans $\mathbb{Q}_{p}\left[G_{F}\right]$, et nous l'appellerons pour simplifier la restriction à $F$ (dans la mesure où elle est aussi issue de la restriction des automorphismes $G_{S} \rightarrow G_{F}$, par prolongement par $\mathbf{Z}_{p}$-linéarité, puis par densité de $\mathbb{Z}_{p}\left[G_{S}\right]$ dans $\wedge_{S}$, et enfin par extension aux éléments de $\Delta_{S}^{\prime}$ ).

(iii) Soit $k_{\infty}=k \mathbb{Q}_{\infty}$ la $\mathbf{Z}_{p}$-extension cyclotomique de $k$; puisque l'on peut supposer la conjecture de Leopoldt satisfaite pour $p$ dans $k$, le $p$-Sylow $A_{S}(p)$ de $A_{S}=$ Gal $\left(k_{S} / k_{\infty}\right)$ est fini ; fixons une décomposition de $G_{S}$ sous la forme $G_{S}=A_{S} \oplus$ $\Gamma, \Gamma \simeq \mathbb{Z}_{p}$; pour $p \neq 2$ il est clair que $\Gamma$ fixe $k\left(\mu_{p}\right)$ et pour $p=2$, on peut toujours choisir $\Gamma$ de telle sorte qu'il fixe $k(\sqrt{-1})\left(\operatorname{car} k_{\infty} n k(\sqrt{-1})=k\right)$, ce que nous supposons.

On désigne alors par $\alpha_{A_{S}}$ la $\mathbf{Z}_{p}$-mesure de Haar sur $A_{S}$ normalisée par la condition que l'intégrale du caractère unité $\chi_{0}$ vérifie $\left\langle\chi_{0}, \alpha_{A_{S}}\right\rangle=\sharp A_{S}(p)$.

Soit enfin $\gamma$ un générateur topologique de $\Gamma$ et soit $T=1-\gamma$. 
(iv) On pose $M_{0}=k\left(\mu_{q}\right), M=k_{\infty} M_{0}=k\left(\mu_{p \infty}\right)$; on définit les caractères continus suivants de $G_{S}$ :

$$
N: G_{S} \rightarrow \mathrm{Gal}(M / k) \rightarrow \mathrm{Gal}\left(\mathbb{Q}\left(\mu_{p^{\infty}}\right) / \mathbb{Q}\right) \rightarrow \mathbb{Z}_{p}^{*}
$$

qui est le caractère de l'action de $G_{S}$ sur $\mu_{p \infty}$ et dont le noyau fixe le corps $M$,

$$
\langle\rangle: G_{S} \stackrel{N}{\rightarrow} \mathbf{Z}_{p} \rightarrow 1+q \mathbf{Z}_{p}, \omega: G_{S} \stackrel{N}{\rightarrow} \mathbf{Z}_{p}^{*} \rightarrow \text { tor }\left(\mathbf{Z}_{p}^{*}\right)
$$

dont les noyaux fixent respectivement $k_{\infty}$ et $M_{0}$. On a $\omega\langle\rangle=N$.

(1.2) Pseudo-mesure de Deligne-Ribet : On sait (cf. [D-R], $[S]$ ) qu'il existe une $\mathbf{Z}_{p}$-pseudo-mesure $D R_{S}$ associée de la façon suivante à la construction des fonctions $L$ $p$-adiques :

Soit $\chi$ un caractère d'ordre fini de $G_{S}$; alors la fonction

$$
L_{S, p}(\chi, s)=\left\langle\chi\langle\rangle^{1-s}, D R_{S}\right\rangle, s \in \mathbf{Z}_{p}
$$

est telle que

$$
L_{S, p}(\chi, 1-n)=L_{S}\left(\chi \omega^{-n}, 1-n\right), \text { pour tout } n \geq 1,
$$

où, en posant $\chi(\mathfrak{A})=\chi\left(\left(\frac{k_{S} / k}{\mathfrak{A}}\right)\right)$ pour tout idéal entier $\mathfrak{A}$ de $k$ étranger à $S, L_{S}$ est la fonction de variable complexe définie par :

$$
\begin{aligned}
L_{S}(\chi, s) & =\sum_{\mathfrak{A}} \chi(\mathfrak{A}) N \mathfrak{A}^{-\boldsymbol{s}} \\
& =L(\chi, s) \prod_{\mathfrak{L} \in S-S_{\infty}}\left(1-\chi(\mathfrak{L}) N \mathfrak{L}^{-\boldsymbol{s}}\right) .
\end{aligned}
$$

(1.2.3) Remarque : La notation $L_{S}$ rappelle que $\chi$ est vu comme caractère de Dirichlet modulo $S$ et donc que la fonction $L_{S}$ considérée peut ne pas être primitive.

On a alors $[S,(1.15)]$ :

$$
D R_{S}=c_{S} \alpha_{A_{S}} T^{-1}+\mu_{S}, c_{S} \in \mathbf{Z}_{p}, \mu_{S} \in \wedge_{S}
$$

De plus, le résultat de $[\mathrm{C}]$ montre, de façon essentielle, que $c_{S}$ est une unité $p$-adique (résultat qui avait été conjecturé par J. Coates).

(1.3) Transformée de Mellin d'ordre $n \geq 1$ :

L'application continue de $G_{S}$ dans $\mathbf{Z}_{p}\left[G_{S}\right]$ qui à $\sigma \in G_{S}$ associe

$$
m^{(n)}(\sigma)=(N \sigma)^{n} \sigma^{-1}
$$

se prolonge par $\mathbf{Z}_{p}$-linéarité puis par densité en une involution de $\wedge_{S}$ qui se prolonge donc à $\Delta_{S}$ (mais on notera que ce n'est pas une involution sur $\Delta_{S}^{\prime}$ ).

On a alors le résultat suivant :

(1.3.1) Lemme : Pour tout $F \subset k_{S},[F: k]<\infty$, et pour tout $n \geq 1$, on a $S t_{S, F}^{(n)}=$ $\left(m^{(n)}\left(D R_{S}\right)\right)_{F}$. 
On a, pour tout caractère $\psi$ de $G_{S}$ qui se factorise modulo Gal $\left(k_{S} / F\right)$ (i.e. pour tout caractère de $F$ ) :

$$
\begin{aligned}
\left\langle\psi, S t_{S, F}^{(n)}\right\rangle & =\sum_{\sigma_{F} \in G_{F}} \zeta_{S, F}\left(\sigma_{F}, 1-n\right) \psi^{-1}\left(\sigma_{F}\right) \quad(\mathrm{cf} .(0.1)) \\
& =L_{S}\left(\psi^{-1}, 1-n\right)
\end{aligned}
$$

or on a (cf. $(1.2 .2))$ :

$$
L_{S}\left(\psi^{-1}, 1-n\right)=L_{S, p}\left(\psi^{-1} \omega^{n}, 1-n\right)
$$

ce qui s'écrit, par (1.2.1) :

$$
\left\langle\psi, S t_{S, F}^{(n)}\right\rangle=\left\langle\psi^{-1} \omega^{n}\langle\rangle^{n}, D R_{S}\right\rangle=\left\langle\psi^{-1} N^{n}, D R_{S}\right\rangle
$$

l'identité suivante :

$$
\left\langle\psi^{-1} N^{n}, \sigma\right\rangle=\left\langle\psi, m^{(n)}(\sigma)\right\rangle \text { pour tout } \sigma \in G_{S}
$$

montre que l'on a :

$$
\left\langle\psi, S t_{S, F}^{(n)}\right\rangle=\left\langle\psi, m^{(n)}\left(D R_{S}\right)\right\rangle
$$

pour tout caractère $\psi$ de $F$; il en résulte, dans $\mathbb{Q}_{p}\left[G_{F}\right]$, l'égalité

$$
S t_{S, F}^{(n)}=\left(m^{(n)}\left(D R_{S}\right)\right)_{F} .
$$

Ceci prouve que $\left(S t_{S, F}^{(n)}\right)_{F}$ est une $\mathbf{Z}_{p}$-distribution sur $G_{S}$ qui appartient à $\Delta_{S}^{\prime}$ (on peut le vérifier directement à partir des propriétés des $\left.S t_{S, F}^{(n)}\right)$.

Nous notons $S t_{S}^{(n)}$ cette distribution, notation qui est cohérente car la restriction de $S t_{S}^{(n)}$ à $F$ est bien $S t_{S, F}^{(n)}$ au sens de (0.1). La mesure $\delta^{(n)}=m^{(n)}(T)$ en est un dénominateur dont les restrictions $\delta_{F}^{(n)}$ sont non nulles et non diviseurs de 0 dans $\mathbf{Z}_{p}\left[G_{F}\right]$ (cf. (2.7) ci-après).

(1.3.2) Corollaire : Les dénominateurs des $\zeta_{S, F}\left(\sigma_{F}, 1-n\right)$ sont les mêmes que ceux de la restriction à $F$ du terme polaire $m^{(n)}\left(c_{S} \alpha_{A_{S}} T^{-1}\right)$ de $S t_{S}^{(n)}$, écrit sur la base canonique de $\mathbb{Q}_{p}\left[G_{F}\right]$ (cf. (1.2.4)).

2) Calcul du terme polaire :

Posons

$$
\pi_{S}^{(n)}=m^{(n)}\left(\alpha_{A_{S}} T^{-1}\right)
$$

on est amené à calculer $\pi_{S, F}^{(n)}$.

A partir des définitions (1.1), (iii) et (iv), précisons quelques notations :

(2.1) Notations :

(i) On pose $\left[k_{\cap} \mathbb{Q}_{\infty}: \mathbb{Q}\right]=p^{n_{p}(k)}$; l'image de $G_{S}$ par le caractère \langle\rangle est alors $1+q p^{n_{p}(k)} \mathbf{Z}_{p}$. 
(ii) Soit $M_{0}^{(n)}$ le sous-corps de $M_{0}=k\left(\mu_{q}\right)$ fixe par le noyau de $\omega^{n}$ et soit $M^{(n)}=$ $k_{\infty} M_{0}^{(n)}$.

(iii) On pose $B_{S}^{(n)}=\mathrm{Gal}\left(k_{S} / M^{(n)}\right)$ et $H^{(n)}=\mathrm{Gal}\left(M^{(n)} / k_{\infty}\right)$.

(iv) On pose enfin $F^{(n)}=M^{(n)} \cap F, G_{F}^{(n)}=\operatorname{Gal}\left(F / F^{(n)}\right)$.

On a $m^{(n)}(T)=1-(N \gamma)^{n} \gamma^{-1}=1-\langle\gamma\rangle^{n} \gamma^{-1}$ puisque $\Gamma$ fixe $M_{0}$ (cf. (1.1), (iii)) et donc que $\omega^{n}(\gamma)=1$, et par conséquent, en posant

$$
\delta^{(n)}=1-\langle\gamma\rangle^{n} \gamma^{-1}
$$

il vient

$$
\left(m^{(n)}(T)\right)_{F}=\delta_{F}^{(n)}=1-\langle\gamma\rangle^{n} \gamma_{F}^{-1}
$$

Etudions maintenant $m^{(n)}\left(\alpha_{A_{S}}\right)$ en écrivant

$$
\alpha_{A_{S}} \sim \alpha_{B_{S}^{(n)}} \sum_{h \in H^{(n)}} h^{\prime}
$$

où $h^{\prime}$ désigne un prolongement arbitraire de $h \in H^{(n)}$ dans $A_{S}$.

On a donc, puisque la restriction à $B_{S}^{(n)}$ du caractère $N^{n}$ est le caractère unité,

$$
m^{(n)}\left(\alpha_{A_{S}}\right) \sim \alpha_{B_{S}^{(n)}} \beta^{(n)}
$$

où

$$
\beta^{(n)}=\sum_{h \in H^{(n)}} \omega^{n}(h) h^{\prime-1} .
$$

Ceci conduit à (cf. (2.3), (2.4)) :

$$
\pi_{S, F}^{(n)} \sim \frac{1}{\delta_{F}^{(n)}} \alpha_{B_{S}^{(n)}, F} \beta_{F}^{(n)}
$$

(2.6) Définition : On pose

$$
\theta_{F}^{(n)}=\sum_{i=0}^{p^{r}-1}\langle\gamma\rangle^{n i} \gamma_{F}^{-i}
$$

où $p^{r}, r \geq 0$, est l'ordre de $\gamma_{F}$ dans $G_{F}$.

Ceci étant, on a :

$$
\begin{aligned}
\delta_{F}^{(n)} \theta_{F}^{(n)} & =\left(1-\langle\gamma\rangle^{n} \gamma_{F}^{-1}\right) \theta_{F}^{(n)} \\
& =1-\langle\gamma\rangle^{n p^{r}} \\
& \sim n q p^{n_{p}(k)+r}
\end{aligned}
$$

(2.7) Remarque : Ceci montre que $\delta_{F}^{(n)}\left(\right.$ et $\left.\theta_{F}^{(n)}\right)$ sont non nuls et non diviseurs de 0 dans $\mathbf{Z}_{p}\left[G_{F}\right]$ et confirme l'appartenance de $S t_{S}^{(n)}$ à $\Delta_{S}^{\prime}$ (cf. (1.1), (i)) ainsi que l'existence de $\left(m^{(n)}\left(D R_{S}\right)\right)_{F}$. 
On étudie maintenant $\pi_{S, F}^{(n)}$ sous la forme issue de (2.5), compte-tenu de (2.6) :

$$
\pi_{S, F}^{(n)} \sim \frac{1}{n q p^{n_{p}(k)+r}} \alpha_{B_{S}^{(n)}, F} \beta_{F}^{(n)} \theta_{F}^{(n)} .
$$

(2.9) Lemme : On a $\pi_{S, F}^{(n)} \sim c_{F}^{(n)} \alpha_{G_{F}^{(n)}} \beta_{F}^{(n)} \theta_{F}^{(n)}$, où

$$
\left.c_{F}^{(n)} \sim \frac{1}{n p^{r} \sharp H^{(n)} \sharp G_{F}^{(n)}} \rho_{S, p}(k) \quad \text { (cf. }(0.2),(\text { iii })\right) .
$$

En effet, $\alpha_{B_{s}^{(n)}, F}$ est une mesure sur $G_{F}^{(n)}$ invariante par translation ; elle est donc de la forme $c \alpha_{G_{F}^{(n)}}, c \in \mathbb{Q}_{p}$, et $c$ se calcule en écrivant que

$$
\left\langle\chi_{0}, \alpha_{B_{S}^{(n)}}\right\rangle=c\left\langle\chi_{0}, \alpha_{G_{F}^{(n)}}\right\rangle
$$

d'où

$$
c=\frac{\sharp B_{S}^{(n)}(p)}{\sharp G_{F}^{(n)}(p)}=\frac{\sharp A_{S}(p)}{\sharp H^{(n)}(p) \sharp G_{F}^{(n)}(p)} .
$$

Or on a

$$
\begin{aligned}
\sharp A_{S}(p) & \sim q p^{n_{p}(k)} \frac{h(k) R_{p}(k)}{2 \sqrt{D(k)}} \prod_{\mathfrak{L} \in S}\left(1-N \mathfrak{L}^{-1}\right) \\
& \sim q p^{n_{p}(k)} \rho_{S, p}(k) \quad(\mathrm{cf} . \quad(0.2),(\mathrm{iii})) ;
\end{aligned}
$$

d'où l'expression de $c_{F}^{(n)} \sim \frac{c}{n q p^{n} p(k)+r}$.

(2.10) Notations : Pour simplifier les notations nous posons

$$
\alpha_{G_{F}^{(n)}}=\alpha_{F}^{(n)}
$$

(mesure de Haar sur $G_{F}^{(n)}=\mathrm{Gal}\left(F / F^{(n)}\right)$ ).

(2.11) Lemme : On a $\alpha_{F}^{(n)} \beta_{F}^{(n)} \neq 0$ si et seulement si $k_{\infty} F^{(n)}=M^{(n)}$.

On a en effet $\alpha_{F}^{(n)} \beta_{F}^{(n)}=\alpha_{F}^{(n)} \sum_{h \in H^{(n)}} \omega^{n}(h) h_{F}^{\prime-1}$ qui est non nul si et seulement si, par restriction à $F^{(n)}$, on a

$$
\beta_{F^{(n)}}^{(n)}=\sum_{h \in H^{(n)}} \omega^{n}(h) h_{F^{(n)}}^{-1} \neq 0,
$$

ce qui a lieu si et seulement si l'application canonique

$$
H^{(n)} \rightarrow \operatorname{Gal}\left(F^{(n)} / k_{\infty n} F^{(n)}\right)
$$

est injective ; d'où la condition. 
(2.11.2) Remarque : Pour $p \neq 2$, la condition équivaut à $M_{0}^{(n)} \subseteq F^{(n)}$ (i.e. $\omega^{n}$ est un caractère de $F$ ) ; pour $p=2$, on a $k_{\infty} F^{(n)}=M^{(n)}$ exactement dans deux cas : le cas où $\omega^{n}$ est un caractère de $F$ et le cas particulier où $M_{0}^{(n)}=k(\sqrt{-1})$ (i.e. $n$ impair) et où $F^{(n)}$ est un corps imaginaire ne contenant pas $\sqrt{-1}$ (ainsi $F^{(n)}$ est un sous-corps de $k\left(\mu_{2 \infty}\right)$ correspondant à un caractère de la forme $\chi \omega, \chi$ caractère de $k_{\infty}$ distinct de $\chi_{0}$ ).

Le cas où $k_{\infty} F^{(n)}$ est distinct de $M^{(n)}$ conduit donc à $\pi_{S, F}^{(n)}=0$, soit $\zeta_{S, F}\left(\sigma_{F}, 1-n\right) \in \mathbf{Z}_{p}$ pour tout $\sigma_{F} \in G_{F}$. On écarte ce cas d'intégralité et on suppose désormais que $k_{\infty} F^{(n)}=$ $M^{(n)}$. On a toujours (cf. (2.9)) :

$$
\pi_{S, F}^{(n)} \sim c_{F}^{(n)} \alpha_{F}^{(n)} \beta_{F}^{(n)} \theta_{F}^{(n)}
$$

les coefficients de $\pi_{S, F}^{(n)}$ dans $\mathbb{Q}_{p}\left[G_{F}\right]$ sont constants sur les classes de $G_{F}$ modulo $G_{F}^{(n)}$ et sont donnés par ceux de la restriction

$$
c_{F}^{(n)} \beta_{F(n)}^{(n)} \theta_{F(n)}^{(n)}
$$

dans $Q_{p}\left[G_{F^{(n)}}\right]$.

On remarque que $M^{(n)}$ est le composé direct sur $k$ de $k_{\infty}$ et de $M_{0}^{(n)} ;$ la remarque (2.11.2) nous permet alors d'envisager 2 cas :

(2.12.1) Cas où $\omega^{n}$ est caractère de $F^{(n)}$ : On a alors

$$
\mathrm{GaI}\left(F^{(n)} / k\right) \simeq \bar{H}^{(n)} \times \bar{\Gamma}
$$

où

$$
\bar{H}^{(n)}=\operatorname{Gal}\left(F^{(n)} / F^{(n)} k_{\infty}\right) \simeq H^{(n)}, \bar{\Gamma}=\mathrm{Gal}\left(F^{(n)} / M_{0}^{(n)}\right) .
$$

Dans ce cas, le groupe $\bar{\Gamma}$ est indépendant de $n$ car $\sharp \bar{\Gamma}=\left[k_{\infty} F: k\right]$ que l'on note $p^{r_{0}}, r_{0} \geq 0$. On a alors (cf. (2.4), (2.6)) :

$$
\begin{aligned}
& \beta_{F^{(n)}}^{(n)}=\sum_{h \in H^{(n)}} \omega^{n}(h) h_{F^{(n)}}^{-1} \in \mathbb{Z}_{p}\left[\bar{H}^{(n)}\right] \\
& \theta_{F^{(n)}}^{(n)}=\sum_{i=0}^{p^{r}-1}\langle\gamma\rangle^{n i} \gamma_{F^{(n)}}^{-i} \in \mathbb{Z}_{p}[\bar{\Gamma}]
\end{aligned}
$$

si $\beta_{F^{(n)}}^{(n)}$ est bien écrit sur la base canonique $\bar{H}^{(n)}$, il n'en est rien pour $\theta_{F^{(n)}}^{(n)}$, et il faut introduire Gal $\left(F / F^{(n)} F_{0}\right)$, où $F_{0}$ est le sous-corps de $F$ fixe par $\gamma_{F}$; on vérifie que $F^{(n)}{ }_{n} F_{0}=M_{0}^{(n)} ;$ on a, en écrivant

$$
\begin{aligned}
i=\lambda p^{r_{0}}+j, \lambda \in\left[0, p^{r-r_{0}}\left[, j \in\left[0, p^{r_{0}}[\right.\right.\right. \\
\begin{aligned}
\theta_{F^{(n)}}^{(n)} & =\sum_{\lambda, j}\langle\gamma\rangle^{n\left(\lambda p^{r_{0}}+j\right)} \gamma_{F^{(n)}}^{-j} \\
& =\sum_{\lambda=0}^{p^{r-r_{0}-1}}\langle\gamma\rangle^{n \lambda p^{r_{0}}} \sum_{j=0}^{p^{r_{0}-1}}\langle\gamma\rangle^{n j} \gamma_{F^{(n)}}^{-j}
\end{aligned}
\end{aligned}
$$


où

$$
\sum_{\lambda=0}^{p^{r-r_{0}-1}}\langle\gamma\rangle^{n \lambda p^{r_{0}}}=\left(\langle\gamma\rangle^{n p^{r_{0}} p^{r-r_{0}}}-1\right)\left(\langle\gamma\rangle^{n p^{r_{0}}}-1\right)^{-1} \sim p^{r-r_{0}}
$$

On obtient alors par (2.12) que les $p$-parties des dénominateurs cherchées sont toutes égales à celle du dénominateur de $c_{F}^{(n)} p^{r-r_{0}}$, donc (cf. (2.9)) de :

$$
\frac{1}{n p^{r_{0}} \sharp H^{(n)} \sharp G_{F}^{(n)}} \rho_{S, p}(k) ;
$$

or $p^{r_{0}} \sharp H^{(n)} \sharp G_{F}^{(n)}=[F: k]$, ce qui conduit au coefficient

$$
\frac{1}{n[F: k]} \rho_{S, p}(k)
$$

D'où l'expression (i) du théorème (0.3).

En effectuant les calculs précédents modulo $\mathbb{Z}_{p}\left(p \in S_{0}\right)$, on obtient le résultat plus précis suivant :

(2.12.1') Proposition : Soit $\sigma \in G_{S}$ et soit $n \geq 1$ tel que $\omega^{n}$ soit un caractère de $F$; alors, pour la restriction $\sigma_{F}$ de $\sigma \grave{a} F$, on a :

$$
\zeta_{S, F}\left(\sigma_{F}, 1-n\right) \equiv \frac{\left[F: F^{(n)}\right] p^{-v_{p}\left(\left[F: F^{(n)}\right]\right)}}{[F: k]} \frac{\log \left(1+q p^{n_{p}(k)+r_{0}}\right)}{1-\left(1+q p^{n_{p}(k)+r_{0}}\right)^{n}} N^{n}(\sigma) \rho_{S, p}(k) \bmod \mathbf{Z}_{p}
$$

(cf. (0.2), (iii) et $(0.3),(\mathrm{i}))$, où $p^{r_{0}}=\left[F_{n} k_{\infty}: k\right]$.

(2.12.2) Cas particulier du cas $p=2$ : C'est le cas (cf. (2.11.2)) où $F^{(n)}$ est imaginaire cyclique de degré noté $2^{r_{0}+1}$ sur $k$, distinct de $k(\sqrt{-1})$, et $n \equiv 1$ modulo 2 ; on a :

$$
\beta_{F^{(n)}}^{(n)}=1-h_{F^{(n)}}
$$

où $h$ est le générateur de $H^{(n)}$,

$$
\theta_{F^{(n)}}^{(n)}=\sum_{i=0}^{2^{r}-1}\langle\gamma\rangle^{n i} \gamma_{F^{(n)}}^{-i}
$$

Posons pour simplifier $\gamma_{F^{(n)}}=\tau$ (d'ordre $2^{r_{0}+1}$ ) ; on a

$$
h_{F(n)}=\tau^{2^{r_{0}}}
$$

et

$$
\begin{aligned}
\beta_{F^{(n)}}^{(n)} \theta_{F^{(n)}}^{(n)} & =\left(1-\tau^{2^{r_{0}}}\right) \sum_{i=0}^{2^{r}-1}\langle\gamma\rangle^{n i} \tau^{-i} \\
& =\sum_{i=0}^{2^{r}-1}\langle\gamma\rangle^{n i} \tau^{-i}-\sum_{i=0}^{2^{r}-1}\langle\gamma\rangle^{n i} \tau^{-i-2^{r_{0}}}
\end{aligned}
$$


Posons :

$$
i=\lambda 2^{r_{0}+1}+j, \lambda \in\left[0,2^{r-r_{0}-1}\left[, j \in\left[0,2^{r_{0}+1}[;\right.\right.\right.
$$

comme $n$ est impair, on peut faire les calculs avec $n=1$. Il vient :

$$
\beta_{F^{(1)}}^{(1)} \theta_{F^{(1)}}^{(1)}=\sum_{\lambda, j}\langle\gamma\rangle^{\lambda 2^{r_{0}+1}+j} \tau^{-j}-\sum_{\lambda, j}\langle\gamma\rangle^{\lambda 2^{r_{0}+1}+j} \tau^{-j-2^{r_{0}}}
$$

dans le second terme, chaque somme sur $j$ s'écrit :

$$
\begin{aligned}
& -\sum_{j=0}^{2^{r_{0}}-1}\langle\gamma\rangle^{\lambda 2^{r_{0}+1}+j} \tau^{-j-2^{r_{0}}}-\sum_{j=2^{r_{0}}}^{2^{r_{0}+1}-1}\langle\gamma\rangle^{\lambda 2^{r_{0}+1}+j} \tau^{-j-2^{r_{0}}} \\
& =-\sum_{k=2^{r_{0}}}^{2^{r_{0}+1}-1}\langle\gamma\rangle^{\lambda 2^{r_{0}+1}+k-2^{r_{0}}} \tau^{-k}-\sum_{k=0}^{2^{r_{0}}-1}\langle\gamma\rangle^{\lambda 2^{r_{0}+1}+k+2^{r_{0}}} \tau^{-k} .
\end{aligned}
$$

Les coefficients de $\tau^{-j}$ sont les suivants, où $\varepsilon=1$ (resp. $\varepsilon=-1$ ) si $j \in\left[0,2^{r_{0}}\right.$ [ (resp. $j \in\left[2^{r_{0}}, 2^{r_{0}+1}[):\right.$

$$
\begin{aligned}
& \sum_{\lambda}\langle\gamma\rangle^{\lambda 2^{r_{0}+1}+j}-\sum_{\lambda}\langle\gamma\rangle^{\lambda 2^{r_{0}+1}+j+\varepsilon 2^{r_{0}}} \\
& =\langle\gamma\rangle^{j}\left(1-\langle\gamma\rangle^{\varepsilon 2^{r_{0}}}\right) \sum_{\lambda=0}^{2^{r-r_{0}-1}-1}\langle\gamma\rangle^{\lambda 2^{r_{0}+1}} \\
& =\langle\gamma\rangle^{j}\left(1-\langle\gamma\rangle^{\varepsilon 2^{r_{0}}}\right)\left(\langle\gamma\rangle^{2^{r_{0}+1} 2^{r-r_{0}-1}}-1\right)\left(\langle\gamma\rangle^{2^{r_{0}+1}}-1\right)^{-1} \\
& \sim 2^{n_{2}(k)+r-1} .
\end{aligned}
$$

Ici les 2-parties des dénominateurs sont égales à celle du dénominateur de $c_{F}^{(n)} \times 2^{n_{2}(k)+r+1}$ qui est aussi celui de

$$
\frac{2^{n_{2}(k)+1}}{\sharp H^{(n)} \sharp G_{F}^{(n)}} \rho_{S, p}(k) ;
$$

on a $\sharp H^{(n)}=2, \sharp G_{F}^{(n)}=\left[F: F_{\cap} k\left(\mu_{2^{\infty}}\right)\right]$; ce qui donne le rationnel $p$-adique :

$$
\frac{2^{n_{2}(k)}}{\left[F: F_{\cap} k\left(\mu_{2}\right)\right]} \rho_{S, p}(k),
$$

et conduit au cas (ii) du théorème (0.3).

De même que pour le cas général, on obtient ici :

(2.12.2') Proposition : On suppose que $F^{(1)}=F_{\cap} k\left(\mu_{2 \infty}\right)$ est une extension cyclique imaginaire de $k$ distincte de $k(\sqrt{-1})$; alors pour $\sigma \in G_{S}$ et pour $n \equiv 1 \bmod 2$, on a

$$
\zeta_{S, F}\left(\sigma_{F}, 1-n\right) \equiv \frac{\left[F: F^{(1)}\right] 2^{-v_{2}\left(\left[F: F^{(1)}\right]\right)}}{[F: k]} \frac{\log \left(1+4.2^{n_{2}(k)+r_{0}}\right)}{1+\left(1+4.2^{n_{2}(k)+r_{0}}\right)^{n}} N^{n}(\sigma) \rho_{S, 2}(k) \bmod \mathbf{Z}_{2},
$$


où $2^{r_{0}}=\left[f_{\cap} k_{\infty}: k\right]$.

(2.13) Eléments de Stickelberger usuels : Pour $k=\mathbb{Q}$ et $n=1$, considérons les éléments de Stickelberger usuels $S t_{F}=\sum_{\sigma_{F} \in G_{F}} \zeta_{F}\left(\sigma_{F}, 0\right) \sigma_{F}^{-1} ;$ ici $S t_{F}$ est primitif, autrement dit on prend pour $S_{0}$ exactement l'ensemble des diviseurs premiers du conducteur $f$ de $F / Q$. On sait alors que

$$
S t_{F}=\sum_{a \in[1, f]^{\prime}}\left(\frac{1}{2}-\frac{a}{f}\right) \sigma_{a, F}^{-1}
$$

où $[1, f]^{\prime}=\{a \in[1, f],(a, f)=1\}$ et où $\sigma_{a}$ est l'automorphisme défini sur $\mathbb{Q}\left(\mu_{f}\right)$ par $\sigma_{a}(\xi)=\xi^{a}$ pour tout $\xi \in \mu_{f}$.

Comme $2 f S t_{F} \in \mathbb{Z}\left[G_{F}\right]$, on va pouvoir donner, quel que soit $p$ premier, la $p$-partie des dénominateurs des coefficients de $S t_{F}$, car le seul cas qui puisse poser problème est le cas $p=2$ lorsque $2 \notin S_{0}$, mais dans ce cas, la partie polaire en 2 est

$$
\frac{1}{2} \sum_{a \in[1, f]^{\prime}} \sigma_{a, F}^{-1} \sim \frac{1}{2}\left[\mathbb{Q}\left(\mu_{f}\right): F\right] \alpha_{F},
$$

où $\alpha_{F}$ est la mesure de Haar sur $G_{F}$, et le calcul du dénominateur est immédiat.

On obtient donc le résultat suivant :

(2.13.1) Corollaire : Soit $F$ une extension abélienne de $\mathbb{Q}$ de conducteur $f$, et pour tout $p$ premier, soit $V_{p}(F, 0)$ la valuation $p$-adique des dénominateurs des fonctions zêta partielles primitives $\zeta_{F}\left(\sigma_{F}, 0\right), \sigma_{F} \in \mathrm{Gal}(F / \mathbb{Q})$; les nombres $V_{p}(F, 0)$ sont les suivants :

(i) $p \nmid 2 f$ : on a $V_{p}(F, 0)=0$;

(ii) $p \mid f$ et $\omega$ est caractère de $F$ (qui est donc imaginaire) : on a

$$
V_{p}(F, 0)=\operatorname{Max}\left(0, v_{p}(p[F: \mathbb{Q}])-\sum_{\ell \mid f} v_{p}(\ell-1)\right) ;
$$

(iii) $p \mid f$ et $\omega$ n'est pas caractère de $F$ : on a $V_{p}(F, 0)=0$ sauf dans le cas où $p=2$ et $F$ contient un sous-corps imaginaire de $\mathbb{Q}\left(\mu_{2^{\infty}}\right)$, auquel cas on a

$$
V_{2}(F, 0)=\operatorname{Max}\left(0, v_{2}\left(\left[F: F_{\cap} Q_{\infty}\right]\right)-\sum_{\ell \mid f} v_{2}(\ell-1)\right) ;
$$

(iv) $p=2, p \nmid f:$ on a

$$
V_{2}(F, 0)=\operatorname{Max}\left(0, v_{2}(2[F: \mathbb{Q}])-\sum_{\ell \mid f} v_{2}(\ell-1)\right) .
$$

(2.13.2) Remarque : Si $F$ est réel, $F \neq \mathbb{Q}$, on sait que $S t_{F}=0$ et ce qui précède (notamment le point (iv) du corollaire) conduit bien à $V_{2}(F, 0)=0$. Si $F=\mathbb{Q}$, on sait que 
$\zeta_{Q}(0)=-\frac{1}{2}\left(S t_{\Theta}=\sum_{a \in[1,1]}\left(\frac{1}{2}-\frac{a}{1}\right)=\frac{1}{2}-\frac{1}{1}=-\frac{1}{2}\right)$, et le point (iv) du corollaire conduit bien à $V_{2}(\mathbb{Q}, 0)=1$.

3) Conjecture de Leopoldt et fonctions zêta partielles : Les résultats précédents nous permettent de donner des critères analytiques précis d'exactitude de la conjecture de Leopoldt pour $p$ dans $k$, critères qui complètent certaines des remarques faites par J.-P. Serre $[S,(3.17), c, d]$.

Ici on prend $S_{0}=\{p\}, p$ premier arbitraire, auquel cas $S=S_{p \cup} S_{\infty}$; on pose alors :

$$
\begin{aligned}
\rho_{p}(k) & =\rho_{S, p}(k)=\frac{h(k) R_{p}(k)}{2 \sqrt{D(k)}} \prod_{\mathfrak{L} \in S}\left(1-N \mathfrak{L}^{-1}\right) \\
& \sim 2^{[k: \mathbb{Q}]} \frac{h(k) R_{p}(k)}{2 \sqrt{D(k)}} \prod_{\mathfrak{p} \in S_{p}} N \mathfrak{p}^{-1} .
\end{aligned}
$$

(3.1) Théorème : Soit $k$ un corps de nombres totalement réel, et soit $p$ premier :

(i) On a $R_{p}(k) \neq 0$ si et seulement s'il existe $e \geq 0$ tel que la fonction zêta partielle de $k$ :

$$
\zeta_{p, e}(s)=\sum_{\mathfrak{A}, N \mathfrak{A} \equiv \mathbf{1}\left(q p^{e}\right)} N \mathfrak{A}^{-s}
$$

ait une valeur non p-entière en $s=0$.

(ii) Si $R_{p}(k) \neq 0$, alors on a :

$$
v_{p}\left(\zeta_{p, e}(0)\right)=v_{p}\left(\frac{1}{2} \rho_{p}(k)\right)+n_{p}(k)-e<0,
$$

pour tout $e \geq e_{0}=v_{p}\left(p^{n_{p}(k)+1} \frac{1}{2} \rho_{p}(k)\right)$; on a en particulier :

$$
R_{p}(k) \sim 4.2^{-[k: \mathbb{Q}]} \prod_{p \in S_{p}} N \mathfrak{p} \sqrt{D(k)} h(k)^{-1} p^{e-n_{p}(k)} \zeta_{p, e}(0),
$$

pour tout $e \geq e_{0}$.

Démonstration : Prenons pour $F$ le corps $k\left(\mu_{q p^{e}}\right), e \geq n_{p}(k)$, qui est contenu dans le corps $k_{S}$ pour $S=S_{p} \cup S_{\infty}$, et considérons la fonction zêta partielle $\zeta_{S, F}(i d, s)$, notée plus simplement $\zeta_{p, e}(s)$; la fonction $\zeta_{p, e}(s)$ est donc définie par $\sum_{\mathfrak{A}} N \mathfrak{A}^{-\boldsymbol{s}}$, où la somme porte sur les idéaux entiers $\mathfrak{A}$ de $k$ tels que $\left(\frac{F / k}{\mathfrak{A}}\right)=i d$, ce qui équivaut, par restriction à $L=\mathbb{Q}\left(\mu_{q p^{e}}\right), \grave{a}\left(\frac{L / \mathbb{Q}}{N \mathfrak{A}}\right)=i d$; or le groupe d'Artin de $L / \mathbb{Q}$ est précisément :

$$
\left\{(a), a>0, a \equiv 1 \bmod q p^{e}\right\} .
$$

Si $R_{p}(k)=0$, on sait qu'en particulier $\zeta_{p, e}(0)$ est $p$-entière. Si $R_{p}(k) \neq 0, \zeta_{p, e}(s)$ est susceptible du cas (i) de (0.3), et la valuation du dénominateur de $\zeta_{p, e}(0)(n=1)$ est

$$
\operatorname{Max}\left(0, v_{p}\left(\frac{[F: k]}{\rho_{p}(k)}\right)\right)
$$


or $[F: k]=\varphi\left(q p^{e}\right) p^{-n_{p}(k)} \sim q p^{e-1-n_{p}(k)}$, et on obtient :

$$
\operatorname{Max}\left(0, v_{p}\left(\frac{2 p^{e-n_{p}(k)}}{\rho_{p}(k)}\right)\right) .
$$

Il suffit alors d'imposer la relation $\frac{2 p^{e-n_{p}(k)}}{\rho_{p}(k)} \sim p$ (i.e. $\left.e_{0}=v_{p}\left(p^{n_{p}(k)+1} \frac{1}{2} \rho_{p}(k)\right)\right)$ pour définir une valeur $e_{0}$ de $e$ à partir de laquelle $\zeta_{p, e}(0)$ est non $p$-entière ; on a ensuite :

$$
\frac{1}{2} \rho_{p}(k) \sim p^{e-n_{p}(k)} \zeta_{p, e}(0)
$$

pour tout $e \geq e_{0}$.

D'où le fait que la valuation de $\zeta_{p, e}(0)$ soit linéaire strictement décroissante par rapport à $e$, et strictement négative à partir de $e_{0}$.

Ceci précise complètement ce que l'on peut attendre au plan numérique.

Dans une direction en un sens opposée, on peut considérer la situation sujvante :

Soit $\zeta_{p}(k, s)$ la fonction zêta $p$-adique de $k$ (égale à $\zeta_{S, k}(i d, s)$ avec $S=S_{p} \cup S_{\infty}$, selon les notations du $\S 0)$, et soit $m_{p}$ l'ordre du caractère $\omega$ sur $G_{S}\left(m_{p}\right.$ est un diviseur pair de $p-1$ pour $p \neq 2$, et $m_{2}=2$ ) ; on peut alors énoncer :

(3.2) Théorème : Soit $k$ un corps de nombres totalement réel, et soit $p$ premier :

(i) On a $R_{p}(k) \neq 0$ si et seulement s'il existe $e \geq 0$ tel que $\zeta_{p}\left(k, 1-\lambda m_{p} p^{e}\right)$ ne soit pas $p$-entière (condition indépendante du choix de $\lambda>0$ ).

(ii) Si $R_{p}(k) \neq 0$, alors on a :

$$
v_{p}\left(\zeta_{p}\left(k, 1-\lambda m_{p} p^{e}\right)\right)=v_{p}\left(\frac{1}{2} \rho_{p}(k)\right)-e<0
$$

pour tout $e \geq e_{0}=v_{p}\left(p \frac{1}{2} \rho_{p}(k)\right)$; on a en particulier :

$$
R_{p}(k) \sim 4.2^{-[k: \Phi]} \prod_{p \in S_{p}} N \mathfrak{p} \sqrt{D(k)} h(k)^{-1} p^{e} \zeta_{p}\left(k, 1-\lambda m_{p} p^{e}\right),
$$

pour tout $e \geq e_{0}$ et tout $\lambda>0$ étranger à $p$.

Démonstration : Prenons donc $F=k$, supposons $R_{p}(k) \neq 0$ et appliquons le théorème (0.3) avec $n=\lambda m_{p} p^{e}, \lambda>0$ étranger à $p$; alors $\omega^{n}$ est bien caractère de $F$ et la valuation du dénominateur de $\zeta_{p}(k, 1-n)$ est

$$
\operatorname{Max}\left(0, v_{p}\left(\frac{n}{\rho_{p}(k)}\right)\right)=\operatorname{Max}\left(0, e+v_{p}(2)-v_{p}\left(\rho_{p}(k)\right)\right) \text {. }
$$

Ensuite, la valeur minimum $e_{0}$ de $e$ pour laquelle $\zeta_{p}\left(k, 1-\lambda m_{p} p^{e}\right)$ est non $p$-entière vérifie la relation :

$$
e_{0}-v_{p}\left(\frac{1}{2} \rho_{p}(k)\right)=1
$$


d'où $e_{0}$.

On a alors comme attendu :

$$
\frac{1}{2} \rho_{p}(k) \sim p^{e} \zeta_{p}\left(k, 1-\lambda m_{p} p^{e}\right),
$$

pour tout $e \geq e_{0}$, ce qui conduit au point (ii) du théorème.

\section{(3.3) Remarques :}

(i) En vertu de (1.2.2), on a, pour tout $n \equiv 0 \bmod m_{p}$ :

$$
\zeta_{p}(k, 1-n)=\zeta(k, 1-n) \prod_{p \in S_{p}}\left(1-N \mathfrak{p}^{n-1}\right) \sim \zeta(k, 1-n)
$$

on peut donc, dans (3.2), remplacer $\zeta_{p}(k)$ par $\zeta(k)$, ce qui signifie que, en pratique, les calculs de valeurs aux entiers négatifs de $\zeta(k, s)$ peuvent suffire à la détermination de $v_{p}\left(h(k) R_{p}(k)\right)$. En outre, seuls les entiers $n$ de la forme $\lambda m_{p} p^{e}$ peuvent donner des valeurs non $p$-entières pour $\zeta(k, 1-n)$.

(ii) On peut, sans difficulté, énoncer des formes mixtes de (3.1) et (3.2) en faisant intervenir à la fois un corps de rayon $F$ convenable et les valeurs, en des entiers négatifs convenables, des fonctions zêta partielles correspondantes.

(iii) L'énoncé (3.2) est à rapprocher de la proposition 4.2 de [K]. 


\section{Bibliographie}

[B] Barsky, D., Fonctions zêta $p$-adiques d'une classe de rayon des corps de nombres totalement réels, Groupe d'Etudes d'Analyse Ultramétrique, 1977-1978; errata 1978-1979.

[CN] Cassou-Noguès, P., Valeurs aux entiers négatifs des fonctions zêta et fonctions zêta p-adiques, Invent. Math. 51 (1979) 29-59.

[C] Colmez, P., Résidu en $s=1$ des fonctions zêta $p$-adiques, Invent. Math. 91 (1988) 371-389.

[D-R] Deligne, P., Ribet, K.A., Values of abelian $L$-functions at negative integers over totally real fields, Invent. Math. 59 (1980) 227-286.

[K] Kolster, M; Remarks on étale $K$-theory and Leopoldt's conjecture (1992), preprint.

[N] Nguyen Quang Do T., Analogues supérieurs du noyau sauvage (1992), à paraître.

[S] Serre, J.-P., Sur le résidu de la fonction zêta $p$-adique d'un corps de nombres, C.R. Acad. Sci., Série A, Paris 287 (1978) 183-188.

[Si] Siegel, C.L., Über die Fourierschen Koeffizienten von Modulformen, Göttingen Nach. 3 (1970), 15-56.

Georges GRAS

Laboratoire de Mathématiques

URA - CNRS n ${ }^{\circ} 741$

F - 25030 Besançon Cedex 